\title{
Thermodynamic Method for Measuring the B/A Nonlinear Parameter Under High Pressure
}

\author{
Piotr KIEŁCZYŃSKI ${ }^{1)}$, Marek SZALEWSKI ${ }^{1)}$, Andrzej BALCERZAK ${ }^{1)}$, \\ Krzysztof WIEJA ${ }^{1)}$, Aleksander J. ROSTOCKI ${ }^{2)}$, \\ Ryszard M. SIEGOCZYŃSKI ${ }^{2}$ \\ 1) Institute of Fundamental Technological Research \\ Polish Academy of Sciences \\ Pawińskiego 5B, 02-106, Warszawa, Poland \\ e-mail: pkielczy@ippt.pan.pl \\ 2) Warsaw University of Technology \\ Faculty of Physics \\ Koszykowa 75, 00-662 Warszawa, Poland
}

\begin{abstract}
The nonlinearity parameter $B / A$ is a measure of the nonlinearity of the equation of state for a fluid. The nonlinearity parameter $B / A$ is a physical parameter often used in acoustics, from underwater acoustics to biology and medicine. It can provide information about structural properties of the medium, internal pressure and inter-molecular spacing. The thermodynamic method has been applied for determination of $B / A$ parameter in diacylglycerol (DAG) oil as a function of pressure at various temperatures. Isotherms of the density and phase velocity of longitudinal ultrasonic wave as a function of pressure have been measured. Using the thermodynamic method along with measured isotherms of sound speed and density, the nonlinearity parameter $B / A$ (for DAG oil) was evaluated as a function of pressure (up to $220 \mathrm{MPa}$ ) at various temperatures ranging from 20 to $50^{\circ} \mathrm{C}$.
\end{abstract}

Key words: nonlinearity parameter $B / A$, thermodynamic method, high pressure, longitudinal ultrasonic wave velocity.

\section{INTRODUCTION}

The nonlinearity parameter $B / A$ is a measure of the nonlinearity of the equation of state for a fluid. It plays a significant role in acoustics, from underwater acoustics to biology and medicine. The nonlinearity parameter is important because it determines distortion of a finite amplitude wave propagating in the fluid. The parameter $B / A$ determines the nonlinear correction to the velocity due to the influence of nonlinear effects caused by the propagation of finite amplitude wave. Moreover, it can be related to the molecular dynamics of the medium 
and it can provide information about structural properties of medium, internal pressures, clustering, and inter-molecular spacing, etc. Importance of the $B / A$ parameter increases with the development of high-pressure technologies of food processing and preservation.

The parameter $\mathrm{B} / \mathrm{A}$ is very important in medical applications of ultrasound, both in diagnosis and therapy. In diagnostic applications knowledge of B/A is necessary in design and optimization of the ultrasound imaging devices. In therapy it enables to predict the temperature in the tissue during ultrasonic hyperthermia treatment. During nonlinear wave propagation the harmonic frequencies are generated and they are absorbed more quickly than the fundamental one $[1,2]$.

The experimental techniques for the $B / A$ parameter measurement can be classified by the two basic approaches: thermodynamic method and finite-amplitude method. In the first method, $B / A$ is evaluated from the definition using measurements of the sound velocity as a function of pressure and temperature [3-8]. The second method is based on the analysis of waveform distortion due to harmonics generation during wave propagation [9]. Thermodynamic method is more reliable method for measuring the nonlinearity parameter $B / A[10]$.

The aim of this work is to determine the nonlinearity parameter $B / A$ for DAG (diacylglycerol) oil as a function of hydrostatic pressure and temperature by applying the thermodynamic method [3-8], that uses the measured isotherms of the density and sound velocity in DAG oil.

The authors applied thermodynamic method to determine $B / A$ parameter in diacylglycerol (DAG) oil composed of $82 \%$ of DAGs and $18 \%$ of triacylglycerols (TAGs), with a vestigial amount of monoacylglycerols (MAGs) and free fatty acids. The fractions were determined by means of the gas chromatography method. DAG oil is an important compound of fatty food products, e.g. vegetable oils [11] and solid animal fats. DAG oil is also applied in the pharmaceutical industry. The consumption of DAG oil is claimed to have beneficial effects on obesity and weight related disorders [12].

In the thermodynamic method to evaluate the nonlinearity parameter $B / A$ one must know (from experiment) the dependence of the longitudinal ultrasonic wave phase velocity on hydrostatic pressure at various temperatures (isotherms). Moreover, one should measure isotherms of the density of a liquid versus pressure. The measurement of sound speed can be performed relatively easily with high accuracy. This is an advantage of the employed thermodynamic method to evaluate the nonlinearity parameter $B / A$.

The authors have carried out the measurement of isotherms of the longitudinal ultrasonic wave velocity as a function of pressure employing the established laboratory measuring setup. To evaluate the sound velocity in DAG oil, an ultrasonic method that uses cross-correlation method to determine the time-of-flight 
between successive impulses was employed. The density of DAG oil was determined from changes of DAG oil sample volume evaluated by measuring the piston displacement in the high-pressure chamber. Consequently, we evaluated the dependence of the nonlinearity parameter $B / A$ on pressure (from atmospheric up to $220 \mathrm{MPa}$ ) and temperature (from 20 to $50^{\circ} \mathrm{C}$ ).

\section{Theoretical BaCKGround}

Expanding the equation of state $P=P(\rho, s)$, (which links pressure $P$, density $\rho$ and entropy $s$ ) of a liquid into a Taylor series along the isentrope $s=s_{0}$, yields [3]:

$$
P-P_{0}=A\left(\frac{\rho^{\prime}}{\rho_{0}}\right)+\frac{B}{2 !}\left(\frac{\rho^{\prime}}{\rho_{0}}\right)^{2}+\frac{C}{3 !}\left(\frac{\rho^{\prime}}{\rho_{0}}\right)^{3}+\ldots,
$$

where $\rho^{\prime}=\rho-\rho_{0}$ is the excess density, $P$ and $\rho$ are instantaneous pressure and density of the liquid disturbed by the ultrasonic wave propagation, $P_{0}$ and $\rho_{0}$ are their unperturbed (ambient) values, and

$$
\begin{aligned}
& \left.A=\rho_{0}\left(\frac{\partial P}{\partial \rho}\right)_{s}\right]_{\rho=\rho_{0}} \equiv \rho_{0} c_{0}^{2}, \\
& \left.B=\rho_{0}^{2}\left(\frac{\partial^{2} P}{\partial \rho^{2}}\right)_{s}\right]_{\rho=\rho_{0}}, \\
& \left.C=\rho_{0}^{3}\left(\frac{\partial^{3} P}{\partial \rho^{3}}\right)_{s}\right]_{\rho=\rho_{0}},
\end{aligned}
$$

where $s$ is specific entropy, $c_{0}$ is the isentropic small-signal sound speed, the partial derivatives are evaluated at the unperturbed state $\left(\rho_{0}, s_{0}\right)$, what is indicated by the subscript 0 . Subscript " $s$ " refers to a constant entropy process, to which the phenomenon of ultrasound propagation can be attributed.

According to the definitions in Eqs. (2.2) and (2.3), the nonlinearity parameter $B / A$ (that is the ratio of quadratic to linear term in the Taylor series expansion) can be expressed as:

$$
\left.\frac{B}{A}=\frac{\rho_{0}}{c_{0}^{2}}\left(\frac{\partial^{2} P}{\partial \rho^{2}}\right)_{s}\right]_{\rho=\rho_{0}} .
$$

Using the definition of the sound speed $c^{2}=\left(\frac{\partial P}{\partial \rho}\right)_{s}$, the formula $(2.5)$ can be rewritten as:

$$
\left.\frac{B}{A}=2 \rho_{0} c_{0}\left(\frac{\partial c}{\partial P}\right)_{s}\right]_{\rho=\rho_{0}}
$$


Upon expanding the derivative $\left.\left(\frac{\partial c}{\partial P}\right)_{s}\right]_{\rho=\rho_{0}}$ we arrive at the following formula [3]:

$$
\left.\left.B / A=2 \rho_{0} c_{0}\left(\frac{\partial c}{\partial P}\right)_{T}\right]_{\rho=\rho_{0}}+2 c_{0} T \alpha_{p} c_{p}^{-1}\left(\frac{\partial c}{\partial T}\right)_{P}\right]_{\rho=\rho_{0}},
$$

where $\rho_{0}$ is the density of undisturbed medium, $c_{0}$ is the sound velocity for acoustic waves of infinitesimal amplitude, $c$ is the measured sound velocity at given temperature and pressure, $P$ is the pressure, $T$ is the temperature in Kelvin, $\alpha_{p}$ is the isobaric thermal expansion coefficient, $c_{p}$ is the isobaric specific heat capacity.

The contribution to $B / A$ parameter from temperature changes is always smaller than that from pressures changes [3].

\section{Measuring method And Setup}

As can be seen from Eq. (2.7), to determine $B / A$ parameter it is necessary to measure accurately sound velocity in the investigated liquid medium as a function of pressure and temperature.

The measuring setup is presented in Fig. 1 and described in [13, 14]. High pressure was generated in a thick-walled cylinder chamber with a simple piston and Bridgman II sealing system. The piston-cylinder assembly was working with a hydraulic press, driven by a hand-operated pump. The piston displacement was controlled by a digital caliper.

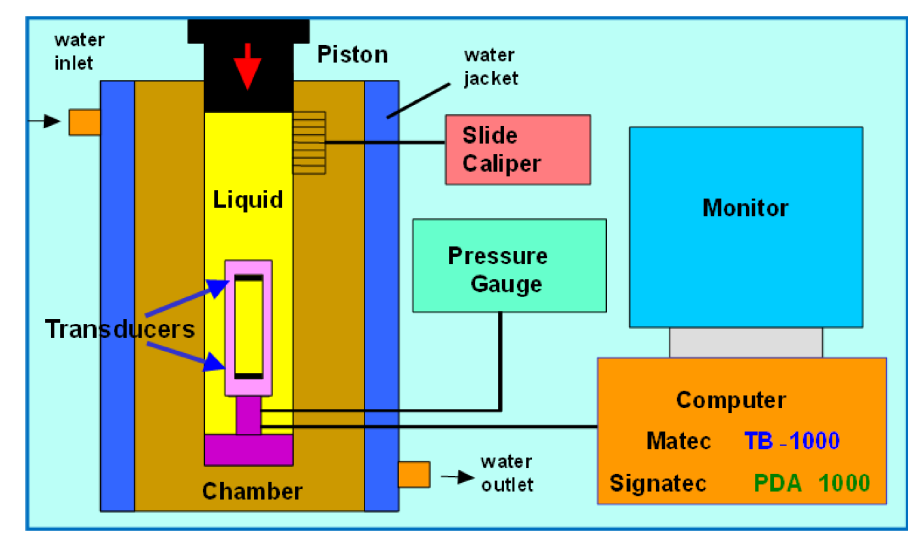

FIG. 1. Experimental ultrasonic setup for measuring the sound velocity in liquids at high pressure and various values of temperature. Temperature of water is stabilized by refrigerated/heating circulator (not presented in the graph).

For pressure measurements a typical $100 \Omega$ manganin transducer was used. Its resistance was measured with a linear unbalanced resistance bridge. The tem- 
perature in the chamber was measured using T-type thermocouple (Cu-constan$\tan )$. A thermostatic bath was circulating in a thermostatic jacket around the chamber. The thermostatic jacket was connected to a precision thermostat. The applied measuring setup and method provided high measurement accuracy.

The accuracy of measurements of ultrasonic wave phase velocity depends on the level of parasitic ultrasonic signals. For measurements of the phase velocity of longitudinal ultrasonic waves, the authors have developed and constructed the computerized setup especially designed to obtain a low level of parasitic ultrasonic signals. A special mounting of transducers in the high-pressure chamber was fabricated. The transducers were operating in the through transmission mode. The transmitting transducer was driven by the TB-1000 pulser - receiver computer card. Ultrasonic pulses ( $5 \mathrm{MHz}$ sine-wave trains) were generated by the transmitting transducer and detected by the receiving transducer. The PDA1000 digitizer card sampled and digitized the signals received by the transducer and amplified by the receiver. The stored signals were then analyzed by computer software.

The transducers were separated by the distance $L$. The time of flight of the ultrasonic pulses was evaluated by applying the cross-correlation method. Measurement of the time of flight of the ultrasonic pulses is difficult and uncertain applying classical methods. Therefore, it is more favorable to use methods based on digital signal processing, e.g. cross-correlation method [15]. The crosscorrelation function $h(t)$, of two functions of time, $f(t)$ and $g(t)$, is defined as follows:

$$
h(t)=\int_{-\infty}^{+\infty} f(\tau) g(t+\tau) \mathrm{d} \tau .
$$

The function $f(t)$ corresponds to the first signal detected by the receiving transducer. This is the ultrasound pulse that travels once across the distance $L$ between the transmitting and receiving transducers. Part of the ultrasonic energy of the first signal is reflected at the receiving transducer back to the transmitting transducer, which in turn reflects part of the incident energy back to the receiving transducer. As a result, the next impulse detected by the receiving transducer (function $g(t)$ ) will travel an extra distance $2 L$ between the transducers. Hence, this signal travels the distance $3 L$. The correlation analysis gives a measure of the similarity between two considered pulses $f(t)$ and $g(t)$ shifted in time. These two pulses have a similar shape but a different amplitude and delay. Therefore, the cross-correlation function reaches a maximum for $t$ equal to the evaluated time difference corresponding to the distance $2 L$. The time of flight was measured with a nanosecond resolution. For each measure- 
ment, the ultrasonic signal was averaged 1024 times in order to improve the signal-to-noise ratio.

The sound velocity was measured with the expanded relative uncertainty equal to $\pm 0.3 \%$ at $95 \%$ confidence level. The expanded relative uncertainty of the density measurements amounts to $\pm 0.05 \%$.

\section{Results}

\subsection{Experimental results and discussion}

4.1.1. Sound velocity. In the DAG oil high-pressure phase transitions occur [16]. As the temperature raises, the pressure at which the phase transition begins increases. At $20^{\circ} \mathrm{C}$ phase transition starts at a pressure of $220 \mathrm{MPa}$, at a temperature of $30^{\circ} \mathrm{C}$ the phase transition starts at a pressure of $300 \mathrm{MPa}$, etc. For this reason, the calculation of nonlinearity parameter value $B / A$ was performed only for the low-pressure phase of DAG oil, in the pressure range from atmospheric pressure up to a pressure of $220 \mathrm{MPa}$. Determination of the parameter of nonlinearity required to carry out measurements of velocity and density isotherms in the DAG oil.

Figure 2 shows the results of longitudinal ultrasonic wave velocity measurements (frequency $5 \mathrm{MHz}$ ) at temperatures: 20, 30, 40, 50 ${ }^{\circ} \mathrm{C}$. Pressure was applied in increments of $10 \mathrm{MPa}$ up to $220 \mathrm{MPa}$. Each increase in pressure was followed by an interval of time ( $5 \mathrm{~min}$ ), which allowed the DAG oil to achieve the thermodynamic equilibrium conditions.

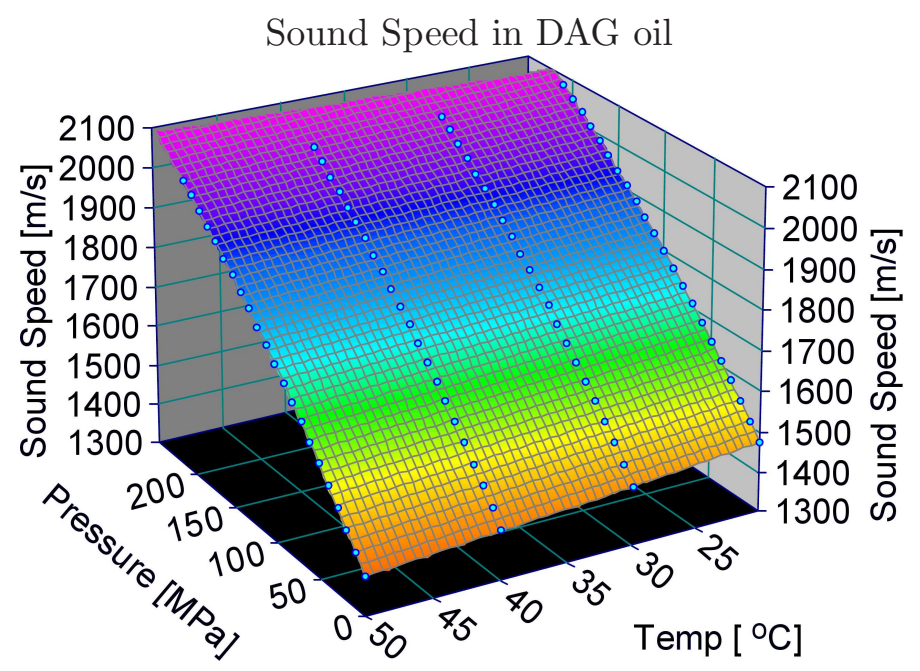

FIG. 2. Plots of sound speed in DAG oil as a function of pressure along various isotherms $\left(T=20,30,40\right.$, and $\left.50^{\circ} \mathrm{C}\right), f=5 \mathrm{MHz}$. 
4.1.2. Density. Figure 3 shows the dependence of the density of DAG oil versus pressure and temperature in the case of low-pressure phase. During experiments, DAG oil volume changes were measured by observation of piston displacement inside the high-pressure chamber. It was measured by a digital caliper gauge. Corrections related to the expansion of the chamber were considered during data analysis. Density values of DAG oil under high pressure were evaluated from the measurements of the DAG oil sample volume, i.e., $\rho(p, T)=m / V(p, T)$, where $m$ is the mass of the sample, and $V(p, T)$ is the sample volume at pressure $p$ and temperature $T$.

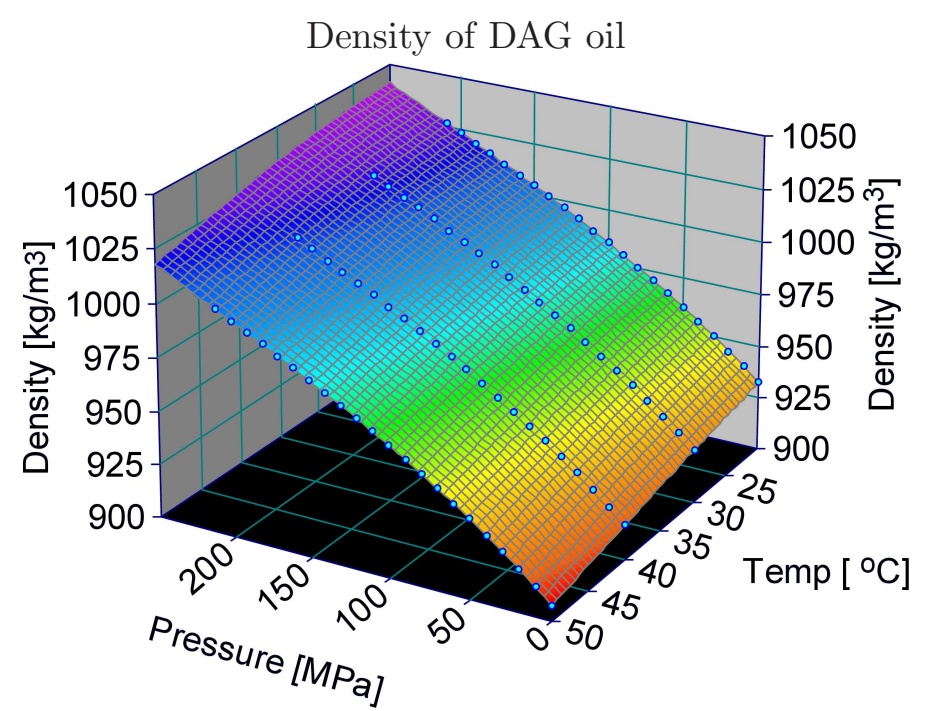

FIG. 3. Density of DAG oil as a function of pressure and temperature.

\subsection{Numerical evaluation of the $B / A$ parameter}

Empirical relationships $c(p, T)$ and $\rho(p, T)$ have been approximated by the appropriate functions of two variables $(p, T)$, i.e., the pressure $p$ and the temperature T. Computer software package Table Curve 3D (Systat, USA) was used to perform the curve fittings. Sound velocity was approximated by using a third order polynomial of two independent variables $(p, T)$ :

$$
c(p, T)=a+b p+c T+d p^{2}+e T^{2}+f p T+g p^{3}+h T^{3}+i p T^{2}+j p T^{2},
$$

where $a=1556.1007, b=3.5558133, c=-5.2591231, d=-0.0046743392$, $e=0.070266476, f=0.0082758464, g=3.8777839 e-6, h=-0.0005704745$, $i=-3.0256387 e-5, j=-9.7492135 e-6$.

Similar approximation was also performed for the density $\rho(p, T)$ of DAG oil. 
4.2.1. Pressure-dependent term $B / A^{\prime}$ of the nonlinearity parameter $B / A$.

$$
\left.B / A^{\prime}=2 \rho_{0} c_{0}\left(\frac{\partial c}{\partial P}\right)_{T}\right]_{\rho=\rho_{0}},
$$

where $\rho_{0}=930.17 \mathrm{~kg} / \mathrm{m}^{3}, c_{0}=1473.30 \mathrm{~m} / \mathrm{s}$.

In order to calculate the dependence of the $B / A^{\prime}$ on the pressure and temperature, one should calculate the derivative of the speed of sound with respect to the pressure at a constant temperature.

4.2.2. The temperature-dependent term $B / A^{\prime \prime}$.

$$
\left.B / A^{\prime \prime}=2 c_{0} T \alpha_{p} c_{p}^{-1}\left(\frac{\partial c}{\partial T}\right)_{P}\right]_{\rho=\rho_{0}} .
$$

Evaluation of the parameter $B / A^{\prime \prime}$ required the calculation of the temperature derivative of the speed of sound. Furthermore, the thermal expansion coefficient $\alpha_{p}$ and specific heat capacity at constant pressure $c_{p}$ had to be calculated, namely:

$$
\alpha_{p}(p, T)=\frac{-1}{\rho(p, T)}\left(\frac{\partial \rho}{\partial T}\right)_{p} .
$$

Specific heat capacity at constant pressure $c_{p}$ has been calculated using the following equation:

$$
c_{p}(p, T)=\frac{T \alpha_{p}^{2}(p, T)}{\rho(p, T)\left(k_{T}-k_{s}\right)},
$$

where $T$ is the temperature in Kelvin, $k_{T}$ is the isothermal compressibility, $k_{s}$ is the adiabatic compressibility.

Isothermal compressibility has been determined from the following formula:

$$
k_{T}(p, T)=\frac{1}{\rho(p, T)}\left(\frac{\partial \rho}{\partial p}\right)_{T} .
$$

Adiabatic compressibility was determined from the expression:

$$
k_{s}(p, T)=\frac{1}{\rho(p, T) c^{2}(p, T)} .
$$


4.2.3. Nonlinearity parameter $B / A$. Using the results of numerical calculations of the pressure-dependent part $B / A^{\prime}$ (from Eq. (4.2)), the temperaturedependent part $B / A^{\prime \prime}$ (from Eq. (4.3)) and the Eq. (2.7), the nonlinearity parameter $\mathrm{B} / \mathrm{A}$ values were evaluated as a function of pressure and temperature.

The plots of calculated values of $B / A$ nonlinear parameter as a function of pressure, at temperatures: $20,30,40,50^{\circ} \mathrm{C}$ are shown in Fig. 4. The derivatives of sound velocity on pressure and temperature were evaluated numerically. $B / A$ nonlinearity parameter has been evaluated in the pressure range before the beginning of the phase transition, for low-pressure phase of DAG oil. The nonlinear parameter $B / A$ depends slightly on the temperature. On the contrary, the pressure dependence of $B / A$ is pronounced. These results are consistent with Beyer's paper [3]. The $B / A$ parameter diminishes quasi-linearly with increasing pressure, see Fig. 4.

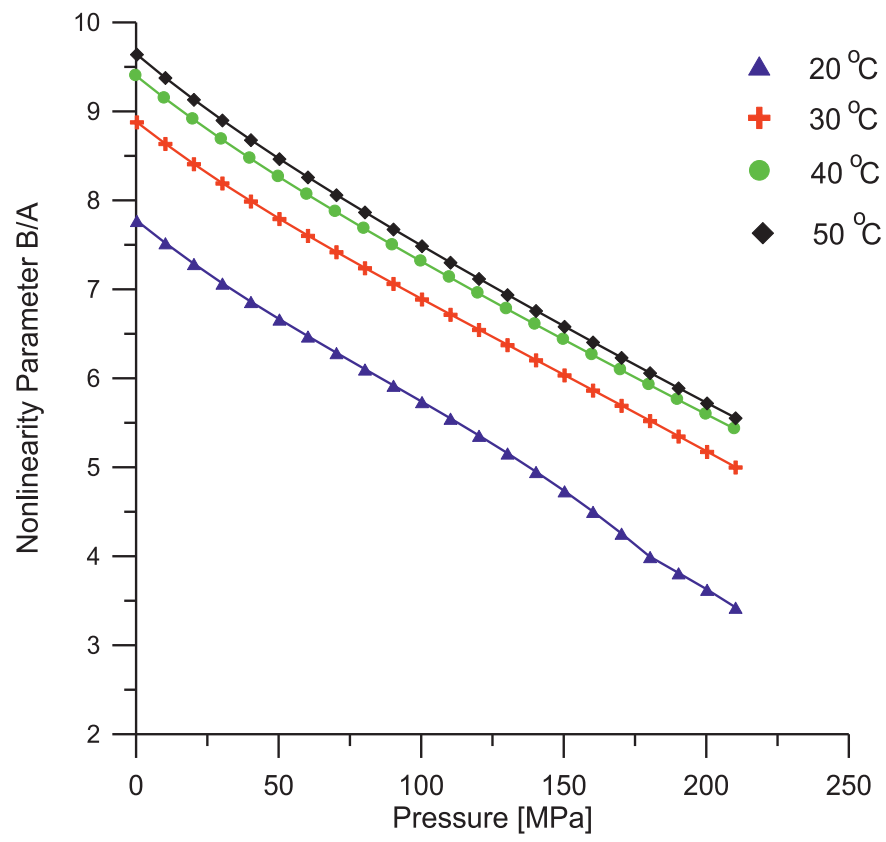

FIG. 4. Nonlinearity parameter $B / A$ as a function of pressure at various temperatures evaluated for the low-pressure phase of DAG oil, $\left(T=20,30,40\right.$, and $\left.50^{\circ} \mathrm{C}\right)$.

The second term $B / A^{\prime \prime}$ in the formula for the parameter of nonlinearity $B / A$ is negative and gives a smaller contribution to the formula for the overall ratio $B / A$ than the pressure-dependent term $B / A^{\prime}$.

To the authors knowledge, evaluation of the $B / A$ nonlinearity parameter in a such broad range of pressures (up to $220 \mathrm{MPa}$ ) is a novelty. 


\section{Conclusions}

Usefulness of ultrasonic thermodynamic method to evaluate the nonlinear parameter $B / A$ that employs the measured velocity and density isotherms versus pressure in the investigated liquid was stated. The performed analysis shows a large monotonic decrease of $B / A$ nonlinearity parameter for DAG oil, with increasing pressure. For example, at temperature $t=50^{\circ} \mathrm{C}, B / A$ nonlinearity parameter decreases from 9.7 at atmospheric pressure, to about 5.6 at $210 \mathrm{MPa}$.

The measuring ultrasonic high-pressure setup and applied procedure have been employed successfully to evaluate the nonlinear parameter $B / A$ for a wide range of pressures and temperatures. The results obtained in this paper can be useful in acoustics, biology, medicine and investigations of materials. Presented in this study method, can also be applied to investigate other liquids, e.g. fuels and biofuels, lubricants, polymers, etc.

Measurements of sound velocity are relatively easy therefore determination of $B / A$ nonlinearity parameter offers a simple way to evaluate the molecular properties of liquids under high pressures at various temperatures. Acoustic nonlinearity is related to the internal pressure, free energy of binding, the effective van der Waals' constants, the translational diffusion coefficient, and the rotational correlation time [17]. This method can be used not only for pure liquids but also for mixtures [18], emulsions and solutions [19].

A number of important thermodynamic parameters of the liquid can be established from measurements of sound speed isotherms as a function of pressure. This will be the subject of future work of the authors.

\section{REFERENCES}

1. Duck F.A., Nonlinear acoustics in diagnostic ultrasound, Ultrasound in Medicine and Biology, 28, 1, 1-18, 2002.

2. Liu X., Gong X., Yin C., Li J., Zhang D., Noninvasive estimation of temperature elevation in biological tissues using acoustic nonlinearity parameter imaging, Ultrasound in Medicine and Biology, 34, 3, 414-424, 2008.

3. Beyer R.T., Parameter of nonlinearity in fluids, J. Acoust. Soc Amer., 32, 719-721, 1960.

4. ZoręBSKI E., ZoręBSKI M., Acoustic nonlinearity parameter B/A determined by means of thermodynamic method under elevated pressure for alkanediols, Ultrasonics, 54, 1, 368$374,2014$.

5. Beyer R.T., The parameter B/A, in Nonlinear Acoustics, M.F. Hamilton and D.T. Blackstock [Eds.], Academic Press, New York, 1998.

6. Khelladi H., Plantier F., Daridon J.L., Djelouah H., Measurement under high pressure of the nonlinearity parameter $B / A$ in glycerol at various temperatures, Ultrasonics, 49, 668-675, 2009. 
7. Coppens A.B., Beyer R.T., Seiden M.B., Donohue J., Guepin F., Hodson R.H., Townsend CH., Parameter of nonlinearity in fluids, Journal of the Acoustical Society of America, 37, 797-804, 1965.

8. Zhu Z., Roos M.S., Cobb W.N., Jensen K., Determination of the acoustic nonlinearity parameter B/A from phase measurements, Journal of the Acoustical Society of America, 87, 5, 797-804, 1983.

9. Adler L., Hiedemann E.A., Determination of the nonlinearity parameter B/A for water and $m$-Xylene, J. Acoust. Soc Amer., 34, 410-412, 1962.

10. LaW W.K., Frizzell L.A., Dunn F., Comparison of thermodynamic and finite amplitude methods of B/A measurement in biological materials, J. Acoust. Soc. Amer., 74, 12951297, 1983

11. Shimizu M., Kudo N., Nakajima N., Matsuo Y., Katsaragi I., Tokimitsu I., Barcelo I., Mateu C., BArcelo F., Acidity and DAG content of olive oil recently produced on the Island of Mallorca, Journal of the American Oil Chemists' Society, 85, 11, 1051-1056, 2008

12. Lo S.K., TAn C.P., Long K., Yusoff M.S.A., Lai O.M., Diacylglycerol oil-properties, processes and products: A review, Food Bioprocess. Technol., 1, 223-233, 2008.

13. Rostocki A.J., Siegoczyński R.M., Kięczyński P., Szalewski M., Balcerzak A., ZDUNIAK M., Employment of a novel ultrasonic method to investigate high pressure phase transitions in oleic acid, High Press. Res., 31, 334-338, 2011.

14. Kielczyński. P., Szalewski M., Rostocki A.J., Zduniak M., Siegoczyński R.M., BALCERZAK A., Investigation of high-pressure phase transitions in vegetable oils by measuring phase velocity of longitudinal ultrasonic waves, IEEE Intern. Ultrason. Symp. Proc., pp. 1563-1566, Rome 2009.

15. Sugasawa S., Time difference measurement of ultrasonic pulses using cross-correlation function between analytic signals, Japanese Journal of Applied Physics, 41, 3299-3307, 2002.

16. Kięczyński P., Szalewski M., Balcerzak A., Malanowski A., SiegoczyńSKI R.M., PtAsZnik S., Investigation of high-pressure phase transitions in DAG (diacylglycerol) oil using the Bleustein-Gulyaev ultrasonic wave method, Food Res. Int., 49, 60-64, 2012.

17. Seghal C.M., Non-linear ultrasonics to determine molecular properties of pure liquids, Ultrasonics, 33, 155-161, 1995.

18. Lu Y., Feng J., Danwu Y., Tong J., Computation of the acoustic nonlinearity parameter in organic liquid binary mixtures, Chinese Science Bull., 45, 414-417, 2000.

19. OAKley B.A., Barber G., Worden T., Hanna D., Ultrasonic parameters as a function of absolute hydrostatic pressure, Journal of Physical and Chemical Reference Data, 32, 4, 1501-1544, 2003.

Received October 28, 2013; revised version February 13, 2014. 